\title{
Weshalb Kinder sich selbst verletzen
}

\author{
Kinder und Jugendliche, die sich ohne suizidale Absicht innerhalb eines Jahres an mindestens \\ fünf Tagen selbst verletzen, zeigen ein nicht-suizidales selbstverletzendes Verhalten (NSSV). \\ Wir sprachen mit dem Kinder- und Jugendpsychiater und Koordinator der neuen S2k-Leitlinie \\ Dr. Paul Plener, Mannheim, u. a. darüber, wie solche Patienten in der Hausarztpraxis auffallen.
}

\section{$?$}

PD Dr. Paul Plener Klinik für Psychiatrie und Psychotherapie des Kindesund Jugendalters Zentrum für Seelische Gesundheit, Mannheim

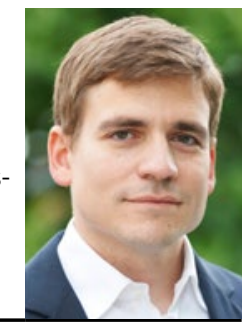

MMW: Was sind die häufigsten Formen von Selbstverletzungen ohne suizidale Absicht und wie verbreitet sind sie?

Plener: Die häufigsten Formen sind Schneiden oder Ritzen, meist an den Unterarmen, im Knöchelbereich oder an den Oberschenkeln. Auch Verbrennungen kommen öfter vor, und mitunter wird auf einen Gegenstand eingeschlagen, bis man blutet. Je nach Studie bejahen ein Viertel bis ein Drittel aller Jugendlichen der Allgemeinbevölkerung in Deutschland ein selbstverletzendes Verhalten in der Vergangenheit, zumindest einmalig. Fragt man nach aktuell vorhandenen wiederholten Selbstverletzungen, sind etwa 4-6\% betroffen.

MMW: Was wird mit der Selbstverletzung erreicht?

Plener: Es funktioniert offensichtlich relativ gut, damit emotional aversive Gefühlslagen für den Moment zu beenden. Wenn sich der Jugendliche angespannt oder schlecht fühlt und sich selbst verletzt, verschwindet das negative Gefühl. Durch die neurobiologische Forschung verstehen wir immer besser, dass Menschen, die sich selbst verletzen, Veränderungen in Gehirnregionen zeigen, die mit Emotionsregulation zu tun haben.

\section{MMW: In welchem Alter beginnen die Selbstverletzungen?}

Plener: Typischerweise beginnen Selbstverletzungen um das zwölfte Lebensjahr. Etwa mit 16 oder 17 Jahren ist ein Höhepunkt erreicht, und zum 18. Lebensjahr hin hören sehr viele dann auf, sich selbst zu verletzen.

\section{MMW: Sind Risikofaktoren bekannt, die ein NSSV fördern?}

Plener: Es gibt den Effekt der „sozialen Ansteckung “. Das Miterleben von Selbstverletzungen stellt einen bedeutenden Risikofaktor dar. Was uns derzeit große Sorgen bereitet, ist, dass soziale Ansteckung nicht nur im realen Leben passiert, sondern auch über soziale Netzwerke und über Bilder und Kommentarfunktionen weitergetragen wird. Außerdem finden wir selbstverletzendes Verhalten gehäuft bei bestimmten Jugendkulturen wie Gothic und Emo. Eine Studie aus dem letzten Jahr hat gezeigt, dass Leute, die sich mit 14 Jahren mit der Gothic Jugendkultur identifizieren, mit 18 ein erhöhtes Risiko für Suizidversuche und -gedanken und selbstverletzendes Verhalten haben. Aber auch Mobbing-Erfahrungen spielen eine riesige Rolle. Deut-

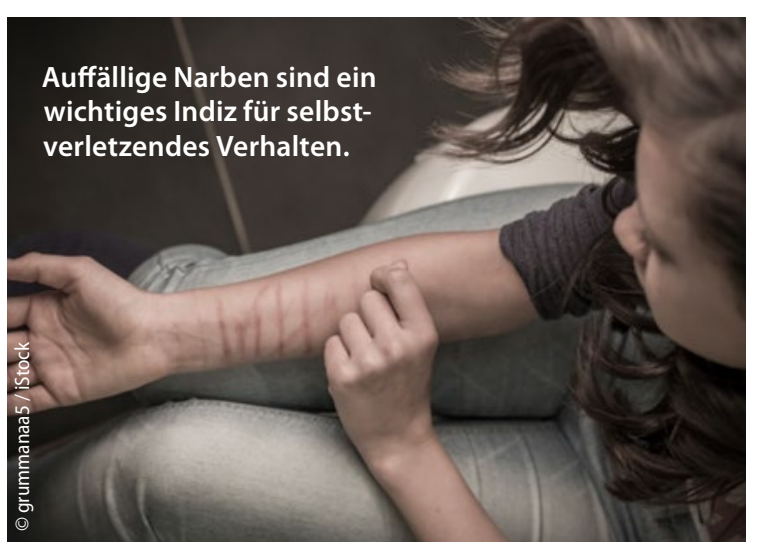

schen Studien zufolge verzwölffacht sich bei häufig gemobbten Jugendlichen das Risiko, sich selbst zu verletzen.

MMW: Wie fallen NSSV-Patienten in der Hausarztpraxis auf?

Plener: Auf typische Narben wird man ja bei der körperlichen Untersuchung aufmerksam. Auch Schnittmuster, für die es kein plausibles Erklärungsmodell gibt, sind auffällig. Abgesehen davon sollten geschilderte depressive sowie Angstsymptome den Hausarzt hellhörig machen.

MMW: Was soll der Hausarzt tun, wenn er den Verdacht auf ein selbstverletzendes Verhalten hegt?

Plener: Eine gute Möglichkeit, damit umzugehen, ist die „respektvolle Neugier“. Auf jeden Fall soll der Hausarzt den Patienten auf das ansprechen, was er gesehen hat. Ein guter Einstieg ist die Frage: „Wobei hilft es dir?“ Damit wird ein Signal gesetzt, dass es hier nicht um Provokation oder Auffallenwollen geht. Für viele Jugendliche, die Zurückweisung erlebt haben oder sich nicht trauen, darüber zu sprechen, ist das ein guter Türöffner. Im Hinblick auf das erhöhte Suizidrisiko dieser Jugendlichen wäre es angebracht zu fragen, ob schon einmal Gedanken an Suizid bestanden haben. $\mathrm{Ob}$ eine Überweisung an einen Kinderund Jugendpsychiater, einen Kinder- und Jugendpsychotherapeuten oder eine Beratungsstelle nötig ist, liegt im Ermessen des ärztlichen Kollegen, indem er ein Gefühl für die Häufigkeit und Akuität der Situation entwickelt.

Interview: Dr. Christine Starostzik 